University of Wollongong

Research Online

Faculty of Engineering and Information

Faculty of Engineering and Information

Sciences - Papers: Part A

Sciences

2016

Highly efficient plasmon excitation in graphene-Bi2Te3 heterostructure

Yao Lu

Soochow University

Jingchao Song

Monash University

Jian Yuan

Soochow University

Lei Zhang

National University of Singapore

Steve Qing Yang Wu

Agency For Science, Technology And Research

See next page for additional authors

Follow this and additional works at: https://ro.uow.edu.au/eispapers

Part of the Engineering Commons, and the Science and Technology Studies Commons

Research Online is the open access institutional repository for the University of Wollongong. For further information contact the UOW Library: research-pubs@uow.edu.au 


\title{
Highly efficient plasmon excitation in graphene-Bi2Te3 heterostructure
}

\author{
Abstract \\ Graphene plasmons have attracted a lot of attention due to large confinement and small mode volume. \\ However, the graphene-based plasmonic devices are still limited in the practical applications due to \\ relatively small light absorption of graphene and limited light-matter coupling efficiency in general \\ excitation strategy. Here, this work reported a strong plasmonic coupling effect observed in a novel \\ graphene- $\mathrm{Bi}_{2} \mathrm{Te}_{3}$ heterostructure on the top of silicon gratings. It is interesting to find that the extinction \\ spectra of the graphene- $\mathrm{Bi}_{2} \mathrm{Te}_{3}$ heterostructure has shown three times greater magnitude than that of \\ graphene. This observation is mainly attributed to two factors: first, the coupling efficiency between the \\ graphene and $\mathrm{Bi}_{2} \mathrm{Te}_{3}$ second, the higher light absorption in the graphene- $\mathrm{Bi}_{2} \mathrm{Te}_{3}$ heterostructure. \\ Moreover, the plasmonic resonance peak of the graphene- $\mathrm{Bi}_{2} \mathrm{Te}_{3}$ heterostructure can be easily tuned by \\ changing the grating period just like what happens in the graphene film. In all, this work utilizes the simple \\ silicon grating to couple the light into the graphene- $\mathrm{Bi}_{2} \mathrm{Te}_{3}$ heterostructure, and further explores the \\ hybridized Dirac plasmons in the graphene- $\mathrm{Bi}_{2} \mathrm{Te}_{3}$ heterostructure. We believe it will stimulate the interest \\ to study the variant plasmonic heterostructure and trigger new terahertz device applications. \\ Disciplines \\ Engineering | Science and Technology Studies

\section{Publication Details} \\ Lu, Y., Song, J., Yuan, J., Zhang, L., Wu, S. Q. Y., Yu, W., Zhao, M., Qiu, C., Teng, J., Loh, K. P., Zhang, C. \& Bao, \\ Q. (2016). Highly efficient plasmon excitation in graphene-Bi2Te3 heterostructure. Journal of the Optical \\ Society of America B: Optical Physics, 33 (9), 1842-1846.

\section{Authors} \\ Yao Lu, Jingchao Song, Jian Yuan, Lei Zhang, Steve Qing Yang Wu, Wenzhi Yu, Meng Zhao, Cheng-Wei Qiu, \\ Jinghua Teng, Kian P. Loh, C Zhang, and Qiaoliang Bao
}




\title{
Highly efficient plasmon excitation in graphene- $\mathrm{Bi}_{2} \mathrm{Te}_{3}$ heterostructure
}

\author{
Yao Lu, ${ }^{1, \dagger}$ Jingchao Song, ${ }^{2, \dagger}$ Jian Yuan, ${ }^{1, \dagger}$ Lei Zhang ${ }^{3}$ Steve Qing Yang Wu, ${ }^{4}$ Wenzhi Yu, ${ }^{1}$ \\ Meng ZhaO, ${ }^{4,5}$ Cheng-Wel Qiu, ${ }^{3}$ Jinghua Teng, ${ }^{4}$ Kian Ping Loh ${ }_{9}^{5}$ Chao Zhang, ${ }^{6}$ And Qiaoliang BaO ${ }^{1,2, *}$ \\ ${ }^{1}$ Institute of Functional Nano and Soft Materials (FUNSOM), Jiangsu Key Laboratory for Carbon-Based Functional Materials and Devices, \\ and Collaborative Innovation Center of Suzhou Nano Science and Technology, Soochow University, Suzhou 215123, China \\ ${ }^{2}$ Department of Materials Science and Engineering, Monash University, Clayton, Victoria 3800, Australia \\ ${ }^{3}$ Department of Electrical and Computer Engineering, National University of Singapore, Singapore 117583, Singapore \\ ${ }^{4}$ Institute of Materials Research and Engineering, Agency for Science, Technology and Research (A*STAR), \\ 3 Research Link, Singapore 117602, Singapore \\ ${ }^{5}$ Department of Chemistry and Graphene Research Centre, National University of Singapore, 3 Science Drive 3, Singapore 117543, Singapore \\ ${ }^{6}$ School of Engineering Physics, University of Wollongong, Wollongong, New South Wales 2552, Australia \\ *Corresponding author: qiaoliang.bao@monash.edu
}

Received 18 May 2016; revised 7 July 2016; accepted 7 July 2016; posted 25 July 2016 (Doc. ID 265378); published 9 August 2016

\begin{abstract}
Graphene plasmons have attracted a lot of attention due to large confinement and small mode volume. However, the graphene-based plasmonic devices are still limited in the practical applications due to relatively small light absorption of graphene and limited light-matter coupling efficiency in general excitation strategy. Here, this work reported a strong plasmonic coupling effect observed in a novel graphene- $\mathrm{Bi}_{2} \mathrm{Te}_{3}$ heterostructure on the top of silicon gratings. It is interesting to find that the extinction spectra of the graphene- $\mathrm{Bi}_{2} \mathbf{T e}_{3}$ heterostructure has shown three times greater magnitude than that of graphene. This observation is mainly attributed to two factors: first, the coupling efficiency between the graphene and $\mathrm{Bi}_{2} \mathrm{Te}_{3}$; second, the higher light absorption in the graphene- $-\mathrm{Bi}_{2} \mathrm{Te}_{3}$ heterostructure. Moreover, the plasmonic resonance peak of the graphene- $\mathrm{Bi}_{2} \mathrm{Te}_{3}$ heterostructure can be easily tuned by changing the grating period just like what happens in the graphene film. In all, this work utilizes the simple silicon grating to couple the light into the graphene- $\mathrm{Bi}_{2} \mathrm{Te}_{3}$ heterostructure, and further explores the hybridized Dirac plasmons in the graphene- $\mathrm{Bi}_{2} \mathbf{T e}_{3}$ heterostructure. We believe it will stimulate the interest to study the variant plasmonic heterostructure and trigger new terahertz device applications. (๑) 2016 Optical Society of America
\end{abstract}

OCIS codes: (250.5403) Plasmonics; (050.2770) Gratings; (160.4236) Nanomaterials.

http://dx.doi.org/10.1364/JOSAB.33.001842

\section{INTRODUCTION}

Surface plasmons (SPs), which are collective oscillations of electrons localized at the conductor/dielectric interface, have been discovered in atomically thin layered materials such as graphene [1]. Due to a number of favorable properties, such as ultrahigh confinement and electrical/chemical tunability, graphene plasmonic structures are widely studied. More importantly, the lifetime of SP modes in graphene can reach hundreds of optical cycles, which potentially circumvents one of the major bottlenecks faced by noble-metal plasmonics $[2,3]$. The graphene surface plasmon polariton has been verified by both infrared transmission spectroscopy and scanning near-field optical microscopy in real-space [4,5]. In order to achieve efficient light-plasmon coupling, Ju et al. explored plasmon excitations by engineering graphene film into microribbon arrays, and found that graphene plasmon resonance peak can be tuned over a broad terahertz frequency range by changing microribbon width and in situ electrostatic doping [6]. However, it is noteworthy that the single-layer graphene supports relatively low resonance frequency/magnitude dependence on carrier concentration. Thus, Yan et al. fabricated graphene/insulator multilayer stacks to effectively enhance the plasmonic magnitude, which is based on the Dirac fermions' carrier density scaling law [7]. Similarly, Zhang et al. used atomically thin hexagonal boron nitride films as spacers to enable strong plasmon coupling between adjacent graphene layers in the vertical dimension, which produces two times upshift of plasmonic resonance frequency and three times enhancement of its magnitude [8].

The surfaces of topological insulators (TIs) and graphene share similar low-energy electronic structures in which the conduction and valence bands touch at Dirac points. Dirac fermions also occur in the two-dimensional (2D) electron gas that 
forms at the surface of TIs as a result of the strong spin-orbit interaction existing in the insulating bulk phase [9]. The spin-plasmons, collective modes originated from the transverse spin fluctuations caused by density fluctuations, have been theoretically predicted in TIs [10]. Pietro et al. reported the first experimental evidence of Dirac plasmon excitations in a TI $\left(\mathrm{Bi}_{2} \mathrm{Se}_{3}\right.$ thin microribbon arrays) [11]. The plasmonic resonance in the $\mathrm{TI}$ is attributed to the bounded oscillation of Dirac quasiparticles of the conducting $2 \mathrm{D}$ edge state. According to theoretical studies [12], the plasmon in graphene will interact and hybridize strongly with itself by inserting a thin dielectric spacer between two layers of graphene to form a "metaldielectric-metal" sandwich structure. Though the graphene and TI plasmons are considered to have the same origin [13], the experimental observation of hybridization between graphene plasmon and other Dirac plasmons has not been reported.

In this work, we fabricated a new graphene- $\mathrm{Bi}_{2} \mathrm{Te}_{3}$ heterostructure by growing $\mathrm{Bi}_{2} \mathrm{Te}_{3}$ on the graphene template. With the help of silicon gratings underneath the heterostructure, the normal incident light can successfully excite the plasmon resonance in the graphene- $\mathrm{Bi}_{2} \mathrm{Te}_{3}$ heterostructure [Fig. 1(a)]. It is interesting to find that the extinction spectra of the graphene$\mathrm{Bi}_{2} \mathrm{Te}_{3}$ heterostructure has shown $3 \times$ greater magnitude of that of the graphene, which is attributed the plasmon coupling between the plasmon at the graphene surface and the top surface of the $\mathrm{Bi}_{2} \mathrm{Te}_{3}$ thin film. Moreover, the extinction peak of the plasmonic resonance of the graphene- $\mathrm{Bi}_{2} \mathrm{Te}_{3}$ heterostructure can be easily tuned in a broad terahertz $(\mathrm{THz})$ frequency range by varying grating periods just like what happens in the graphene film. Since plasmon in graphene and plasmon in $\mathrm{Bi}_{2} \mathrm{Te}_{3}$ share a similar origin, the study of the hybridization between them will stimulate the interest to study variant plasmonic heterostructures and trigger new terahertz device applications.

\section{RESULTS AND DISCUSSION}

$\mathrm{Bi}_{2} \mathrm{Te}_{3}$ is a small bandgap $(\sim 0.145 \mathrm{eV})$ material with rhombohedral crystal structure, in which a sequence of Te-Bi-Te-Bi-Te along the c-axis is followed. Each of the smallest vertically repeated units forms one quintuple layer (QL). It is covalently bonded within the QLs, while attached together via van der Waals force between the QLs. In the $\mathrm{x}-\mathrm{y}$ plane, $\mathrm{Bi}_{2} \mathrm{Te}_{3}$ shares a similar hexagonal lattice with graphene. The periodicity of $\mathrm{Bi}_{2} \mathrm{Te}_{3}$ along graphene carbon atoms is $4.38 \AA$, and the length of graphene $\mathrm{C}-\mathrm{C}$ bond is $1.42 \AA$. Thus, the lattice mismatch between $\mathrm{Bi}_{2} \mathrm{Te}_{3}$ and graphene is $2.7 \%(0.12 \AA)$, which is ideal for van der Waals epitaxial growth [Fig. 1(b)] [14-17]. This work utilizes the chemical vapor deposition (CVD) method to fabricate the graphene- $\mathrm{Bi}_{2} \mathrm{Te}_{3}$ plasmonic heterostructure. First, monolayer graphene film was grown on copper substrates. Then, the $\mathrm{Bi}_{2} \mathrm{Te}_{3}$ nanocrystals were deposited on the graphene film. Subsequently, they were transferred onto silicon grating substrates with the help of a thin poly-(methyl methacrylate) (PMMA) layer. Finally, the PMMA layer was removed via thermal annealing in the vacuum.

The scanning electron microscopy (SEM) images of graphene- $\mathrm{Bi}_{2} \mathrm{Te}_{3}$ heterostructure films with different thicknesses are shown in Figs. 2(a) and 2(b). The reaction times for

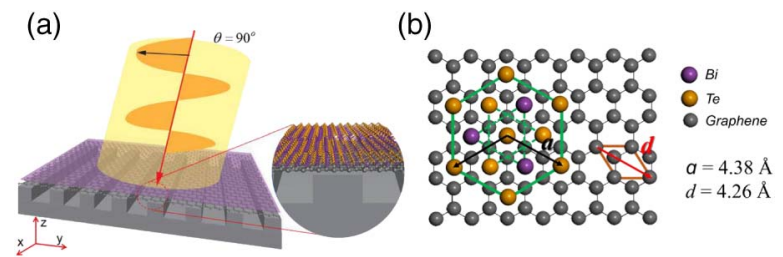

Fig. 1. Schemes showing (a) the graphene- $\mathrm{Bi}_{2} \mathrm{Te}_{3}$ heterostructure on the silicon diffraction grating and (b) lattice mismatch between graphene and $\mathrm{Bi}_{2} \mathrm{Te}_{3}$.
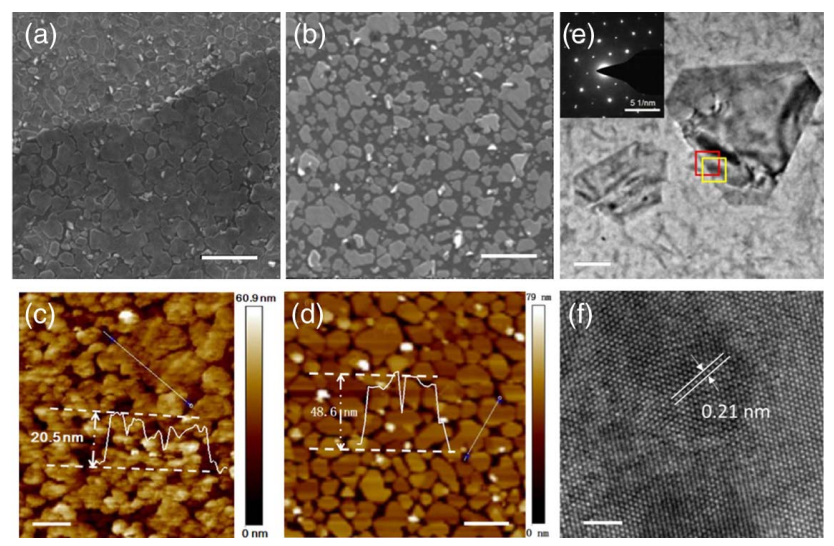

Fig. 2. Characterizations of the graphene- $\mathrm{Bi}_{2} \mathrm{Te}_{3}$ heterostructure. (a),(b) SEM images and (c),(d) AFM images of graphene- $\mathrm{Bi}_{2} \mathrm{Te}_{3}$ heterostructures with different $\mathrm{Bi}_{2} \mathrm{Te}_{3}$ thicknesses. The $\mathrm{Bi}_{2} \mathrm{Te}_{3}$ thicknesses in (a) and (c) are around $20 \mathrm{~nm}$; in (b) and (d) are around $50 \mathrm{~nm}$. Scale bars in (a) and (b) are $2 \mu \mathrm{m}$. Scale bars in (c) and (d) are $1 \mu \mathrm{m}$. (e) TEM image of the graphene- $\mathrm{Bi}_{2} \mathrm{Te}_{3}$ heterostructure. The inset of SAED pattern was taken at the red square area in the TEM image. Scale bar is $1 \mu \mathrm{m}$. (f) HRTEM images taken at the yellow square area in TEM image [Fig. 2(e)]. Scale bar is $2 \mathrm{~nm}$.

growing $\mathrm{Bi}_{2} \mathrm{Te}_{3}$ samples shown in Figs. 2(a) and 2(b) are about $30 \mathrm{~min}$ and $60 \mathrm{~min}$, respectively. The direct consequence of the deposition times is the variation in the $\mathrm{Bi}_{2} \mathrm{Te}_{3}$ thickness. As revealed in the atomic force microscopy (AFM) image [Figs. 2(c) and 2(d)], the thicknesses of $\mathrm{Bi}_{2} \mathrm{Te}_{3}$ in the heterostructure shown in Figs. 2(a) and 2(b) are 20 and $50 \mathrm{~nm}$, respectively. The microstructure of the graphene- $\mathrm{Bi}_{2} \mathrm{Te}_{3}$ heterostructure film was further characterized by transmission electron microscopy (TEM) [Fig. 2(e)]. Both graphene and $\mathrm{Bi}_{2} \mathrm{Te}_{3}$ nanocrystals are revealed, in which the latter shows a quasihexagonal shape. The regular shape and sharp edges indicate an excellent crystallinity of $\mathrm{Bi}_{2} \mathrm{Te}_{3}$ [18]. The selected area electron diffraction (SAED) pattern [inset of Fig. 2(e)] exhibits a clear hexagonal symmetry, which can be indexed to along [001] zone axis of graphene- $\mathrm{Bi}_{2} \mathrm{Te}_{3}$. This further confirms the single crystalline nature of $\mathrm{Bi}_{2} \mathrm{Te}_{3}$. The atomic structure of $\mathrm{Bi}_{2} \mathrm{Te}_{3}$ was characterized using high-resolution transmission electron microscopy (HRTEM) at $200 \mathrm{keV}$. Figure 2(f) clearly resolves the hexagonal lattice fringes. In particular, the lattice fringes are structurally uniform with a spacing of $0.21 \mathrm{~nm}$. It is in good agreement with that of the $(11 \overline{2} 0)$ planes of the rhombohedral phase of $\mathrm{Bi}_{2} \mathrm{Te}_{3}$ [19]. 

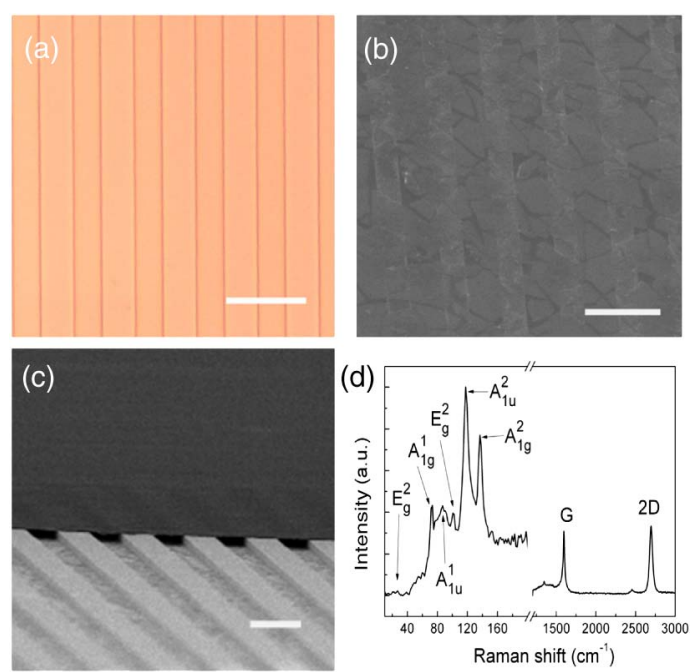

Fig. 3. Fabrication of graphene- $\mathrm{Bi}_{2} \mathrm{Te}_{3}$ plasmonic devices. (a) Optical microscope image of the silicon grating. Scale bar is $10 \mu \mathrm{m}$. (b) SEM image showing the graphene- $\mathrm{Bi}_{2} \mathrm{Te}_{3}$ heterostructure on the Si grating. Scale bar is $10 \mu \mathrm{m}$. (c) Tilted SEM image showing the graphene- $\mathrm{Bi}_{2} \mathrm{Te}_{3}$ heterostructure on a $2 \mu \mathrm{m}$ deep silicon grating. Scale bar is $5 \mu \mathrm{m}$. (d) Raman spectrum of the graphene- $\mathrm{Bi}_{2} \mathrm{Te}_{3}$ heterostructure measured at $633 \mathrm{~nm}$ excitation.

One-dimensional gratings with the different periods (i.e., $4,8,12$, and $16 \mu \mathrm{m}$ ) were fabricated with intrinsic silicon. Figure 3(a) shows an optical microscope image of a silicon grating with a period of $8 \mu \mathrm{m}$. After the wet transfer, PMMAsupported graphene and graphene- $\mathrm{Bi}_{2} \mathrm{Te}_{3}$ films are very well suspended on silicon gratings. The frames of the gratings have been revealed in the SEM image in Fig. 3(b). A clear SEM snapshot was taken from a tilted angle in Fig. 3(c). In order to avoid the mechanical damage made to graphene from solvents, high temperature annealing rather than acetone rinsing was used to remove the PMMA layer. In this way, silicon grating supported graphene and graphene- $\mathrm{Bi}_{2} \mathrm{Te}_{3}$ films are ready for spectroscopy measurements. Raman spectra were measured at room temperature with the excitation of a $633 \mathrm{~nm}$ laser. Sample damage is avoided by controlling the power below $0.5 \mathrm{~mW}$. Figure $3(\mathrm{~d})$ shows a typical Raman spectrum from 20 to $3000 \mathrm{~cm}^{-1}$, which covers the interested spectrum ranges of both the TI and graphene. In the low-frequency range, the Raman spectrum exhibits five characteristic of $\mathrm{Bi}_{2} \mathrm{Te}_{3}$ crystal at $39,69,92,101,117$, and $137 \mathrm{~cm}^{-1}$, which correspond to the $E_{g}^{1}, A_{1 g}^{1}, A_{1 u}^{1}, E_{g}^{2}, A_{1 w}^{2}$, and $A_{1 g}^{2}$ vibrational modes of single-crystalline $\mathrm{Bi}_{2} \mathrm{Te}_{3}$, respectively. At the same time, two characteristic peaks of graphene are observed at $1598 \mathrm{~cm}^{-1}$ (G band) and $2698 \mathrm{~cm}^{-1}$ (2D band). Compared with the Raman spectrum of monolayer graphene, it is interesting to observe the blueshift of the G band and significant intensity reduction of the $2 \mathrm{D}$ band in the graphene- $\mathrm{Bi}_{2} \mathrm{Te}_{3}$ heterostructure. This is also an indication of the doping effect on the graphene film [20].

The plasmon resonance in the graphene- $\mathrm{Bi}_{2} \mathrm{Te}_{3}$ heterostructure was studied using Fourier transform infrared (FTIR) spectroscopy. The extinction spectra of graphene and graphene- $\mathrm{Bi}_{2} \mathrm{Te}_{3}$ on various periods were shown as solid lines in Figs. 4(a) and 4(b). It is found that the plasmon resonance (a)

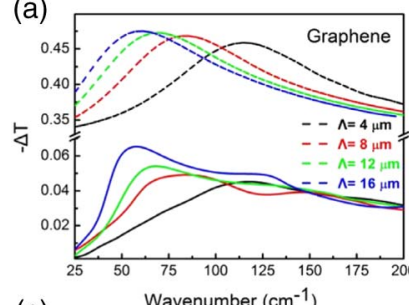

(c)
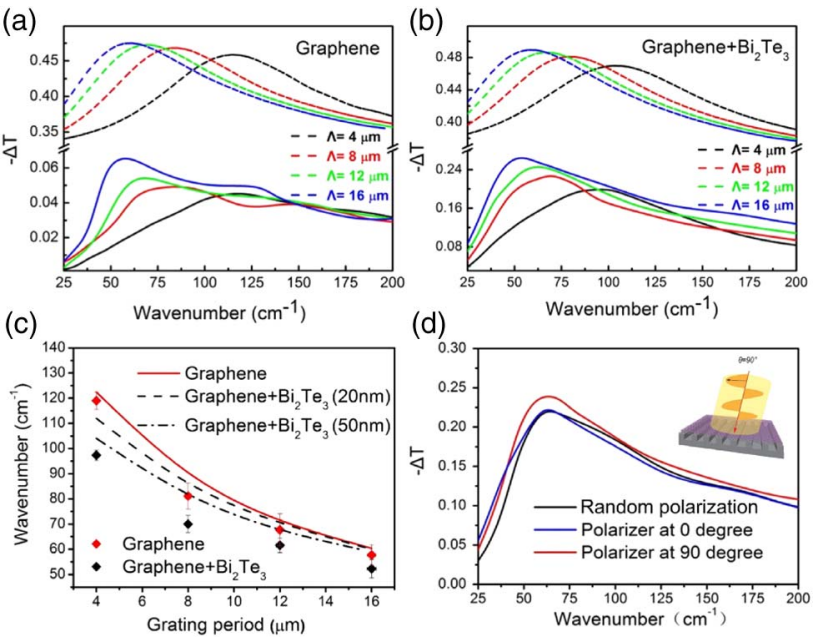

(d)

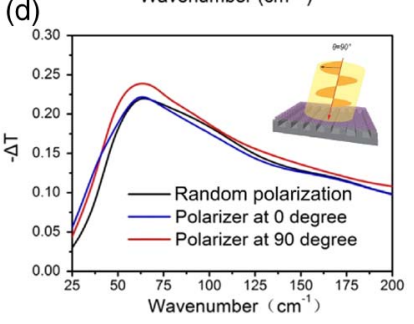

Fig. 4. Experimental and simulated spectra of the graphene and graphene- $\mathrm{Bi}_{2} \mathrm{Te}_{3}$ heterostructures. The experimental extinction spectra of (a) graphene film and (b) graphene- $\mathrm{Bi}_{2} \mathrm{Te}_{3}$ on silicon gratings with different periods, where incident light is vertically polarized to the grating axis. (c) Simulated frequency shift of graphene film (red line) and graphene- $\mathrm{Bi}_{2} \mathrm{Te}_{3}$ heterostructure (black dashed line) with various $\mathrm{Si}$ grating periods. (d) The extinction spectra of graphene- $\mathrm{Bi}_{2} \mathrm{Te}_{3}$ on the $\mathrm{Si}$ grating with polarized incident infrared light. Polarizer at $90 \mathrm{deg}$ refers to the polarization perpendicular to the grating (red trace) and polarizer at 0 deg refers to the polarization parallel to the grating (blue).

peaks for both graphene and graphene- $\mathrm{Bi}_{2} \mathrm{Te}_{3}$ heterostructures shift to lower wavenumbers when the grating periodicity is reduced. In particular, the resonance peak shifts from 57.7 to $118.9 \mathrm{~cm}^{-1}$ when the grating period changes from 16 to $4 \mu \mathrm{m}$ for graphene [Fig. 4(a)]. In the graphene- $\mathrm{Bi}_{2} \mathrm{Te}_{3}$ case, the resonance peak shifts from 52.3 to $97.4 \mathrm{~cm}^{-1}$ when the grating period changes from 16 to $4 \mu \mathrm{m}$ [Fig. 4(b)]. In comparison, the fundamental mode plasmon resonance peak of the graphene- $\mathrm{Bi}_{2} \mathrm{Te}_{3}$ heterostructure blueshifts by $21.5 \mathrm{~cm}^{-1}$ than that of graphene (on a $4 \mu \mathrm{m}$ grating). It has also been noticed that the resonance tuning range of the graphene- $\mathrm{Bi}_{2} \mathrm{Te}_{3}$ is smaller than that of the graphene alone. This is caused by the addition of the more dielectric $\mathrm{Bi}_{2} \mathrm{Te}_{3}$ layer on the top of graphene. Moreover, the resonance of graphene- $\mathrm{Bi}_{2} \mathrm{Te}_{3}$ under similar incident conditions is enhanced by more than three times. This significant change can be concluded by several reasons: first, the additional $\mathrm{Bi}_{2} \mathrm{Te}_{3}$ layer enhances the light absorption in the heterostructure; second, the addition of the $\mathrm{Bi}_{2} \mathrm{Te}_{3}$ layer also introduces the plasmon coupling in graphene and $\mathrm{Bi}_{2} \mathrm{Te}_{3}$ layered structures. These characteristics attest to the strong plasmonic hybridizing effect between graphene and $\mathrm{Bi}_{2} \mathrm{Te}_{3}$.

To better understand the plasmonic resonance in graphene$\mathrm{Bi}_{2} \mathrm{Te}_{3}$ heterostructures, numerical simulation is performed. In the simulation, the dynamic conductivity of the graphene$\mathrm{Bi}_{2} \mathrm{Te}_{3}$ film follows the Drude mode, like graphene [2]. Only the intraband contributions of the spin-plane wave vector for the graphene- $\mathrm{Bi}_{2} \mathrm{Te}_{3}$ heterostructure have been considered. The simulated extinction spectra of the graphene and graphene- $\mathrm{Bi}_{2} \mathrm{Te}_{3}$ heterostructures are shown as dashed lines in 
Figs. 4(a) and 4(b). It is found that the resonance peak position of the graphene and graphene- $\mathrm{Bi}_{2} \mathrm{Te}_{3}$ heterostructures matches well with those in the experiment results. The resonant frequency in both the graphene and graphene- $\mathrm{Bi}_{2} \mathrm{Te}_{3}$ heterostructures increases as the period of the silicon grating decreases, which is consistent with the experimental results. Comparing Fig. 4(a) with Fig. 4(b), it is also noticed that the tuning range of the graphene- $\mathrm{Bi}_{2} \mathrm{Te}_{3}$ heterostructure has been decreased from $61.2 \mathrm{~cm}^{-1}$ (graphene) to $45.1 \mathrm{~cm}^{-1}$ (graphene- $\mathrm{Bi}_{2} \mathrm{Te}_{3}$ heterostructure). This is attributed to the increase of dielectric constants in the graphene- $\mathrm{Bi}_{2} \mathrm{Te}_{3}$ heterostructure. It is also noticed that magnitude of the experimental graphene extinction spectra is much weaker than that of the simulated graphene extinction spectra, which could be explained by the possible defects in the real samples. However, the intensity weakening in the graphene- $\mathrm{Bi}_{2} \mathrm{Te}_{3}$ heterostructure is less obvious than that in graphene, which indicates the robustness of the graphene- $\mathrm{Bi}_{2} \mathrm{Te}_{3}$ heterostructure.

In order to have a complete picture about grating-coupled plasmon resonance in the graphene- $\mathrm{Bi}_{2} \mathrm{Te}_{3}$ heterostructure, we plotted the plasmon resonance of different materials as a function of grating period in Fig. 4(c). The experimental data points were obtained by fitting the experimental spectra in Figs. 4(a) and 4(b) with Lorentzian function. Both simulation and experimental results confirmed that the plasmon resonance in the graphene- $\mathrm{Bi}_{2} \mathrm{Te}_{3}$ heterostructure can be effectively tuned by changing the grating period. While the period of the silicon grating is fixed, the extra $\mathrm{Bi}_{2} \mathrm{Te}_{3}$ layer on graphene will cause redshift in plasmon resonance due to the change of dielectric constant. It is interesting to see that thicker $\mathrm{Bi}_{2} \mathrm{Te}_{3}$ crystals on graphene induces further redshift of the resonance frequency.

Figure 4(d) depicts the extinction spectra $(-\Delta T)$ of the graphene- $\mathrm{Bi}_{2} \mathrm{Te}_{3}$ heterostructure under randomly polarized and linearly polarized illuminations. Under randomly polarized illumination and $0^{\circ}$ polarized illumination (polarization direction parallel to the gratings), both spectra (black line and blue line, respectively) have shown a broad bump with a slight peak at $63 \mathrm{~cm}^{-1}(\sim 1.89 \mathrm{THz})$. While, under $90^{\circ}$ polarized illumination (polarization direction perpendicular to the gratings), the peak at $63 \mathrm{~cm}^{-1}$ is much stronger than that under $0^{\circ}$ polarized illumination. This is because the light perpendicular to the grating axis $\left(\theta=90^{\circ}\right)$ can excite bounded electron oscillation [11].

\section{EXPERIMENTAL RESULTS}

\section{A. Material Synthesis}

Graphene films were grown on $25 \mu \mathrm{m}$ thick copper foils (Alfa Aesar, item no. 13382) in a typical CVD system and transferred onto the silicon grating. The graphene film is p-doped as a mixed solution of $\mathrm{FeCl}_{3}$ and $\mathrm{HCl}$ was used to etch away copper during the transferring process. In order to maintain the integrity of the graphene- $\mathrm{Bi}_{2} \mathrm{Te}_{3}$ heterostructures, $\mathrm{Bi}_{2} \mathrm{Te}_{3}$ nanoplatelets were epitaxially grown on the transferred graphene film on silicon gratings by the physical vapor deposition method in a separate tube furnace. Specifically, high purity $\mathrm{Bi}_{2} \mathrm{Te}_{3}$ powder (Alfa Aesar, purity: 99.999\%) and silicon grating substrate with graphene were placed in the center facing downstream of the furnace, respectively. Then the furnace was heated up to $500^{\circ} \mathrm{C}$ and maintained for 90-300 $s$ with argon (with a gas flow rate of $100 \mathrm{sccm}$ ) as carrier and protective gas to transport $\mathrm{Bi}_{2} \mathrm{Te}_{3} \mathrm{va}-$ por onto the graphene film. Then the furnace was cooled down naturally to room temperature.

\section{B. Material Characterizations}

The morphologies and microstructures of the silicon grating and graphene- $\mathrm{Bi}_{2} \mathrm{Te}_{3}$ heterostructure were investigated by SEM (FEI Quanta 200 FEG, acceleration voltage: 5-30 kV) and TEM (FEI Tecnai F30, acceleration voltage: $200 \mathrm{kV}$ ). Raman spectra were recorded by a micro-Raman system (Horiba JobinYvon, HR800) with an excitation wavelength of $633 \mathrm{~nm}$. The laser spot of the Raman system is focused to $<2 \mu \mathrm{m}$ with $\times 100$ object lens. A Bruker Vertex $80 \mathrm{~V}$ FTIR system covering the far-IR $\left(40-700 \mathrm{~cm}^{-1}\right)$ wavelength range was used to investigate the transmission of the graphene$\mathrm{Bi}_{2} \mathrm{Te}_{3}$ heterostructure. As for the far-IR characterizations, a liquid nitrogen cooled mercury-cadmium-telluride detector and a far-IR deuterated triglycine sulfate detector were used in conjunction with potassium bromide and polyethylene terephthalate beam splitters, respectively. The reference used in the spectroscopy measurement is the silicon grating without the graphene and TI layer.

\section{Fabrication of Silicon Grating}

Standard semiconductor fabrication processes were used to fabricate the grating on an intrinsic silicon wafer. UV lithography was applied to define the grating pattern following with dry etching to obtain the gratings.

\section{Numerical Simulation}

All the numerical simulations were carried out with Lumerical FDTD. Periodic boundary conditions were used along the $\mathrm{x}$ axis and perfect matched layers were used along the z-axis. Linearly polarized light was normally incident on the structures. The refractive index of $\mathrm{Si}$ was set to be 3.42 . The conductivity of single-layer graphene was calculated with Kubo formula [21], where the chemical potential and scattering rate were set to be 0.5 and $0.005 \mathrm{eV}$, respectively, to match the experimental results. A layer of $\mathrm{Bi}_{2} \mathrm{Te}_{3}$ covers the graphene layer with complex refractive index $n=8.1+2.6 \mathrm{i}$ [22].

\section{CONCLUSION}

In summary, we have successfully demonstrated a new robust graphene- $\mathrm{Bi}_{2} \mathrm{Te}_{3}$ heterostructure. This graphene- $\mathrm{Bi}_{2} \mathrm{Te}_{3}$ heterostructure has shown three times greater magnitude of that of the graphene due to higher light absorption and strong graphene and $\mathrm{Bi}_{2} \mathrm{Te}_{3}$ coupling. The resonance peak of the graphene- $\mathrm{Bi}_{2} \mathrm{Te}_{3}$ heterostructure has been proved to be tunable by changing the grating periodicities, theoretically and experimentally. This work opens up new possibilities for the applications of graphene- $\mathrm{Bi}_{2} \mathrm{Te}_{3}$ heterostructures in various terahertz photonic devices.

Funding. A*STAR Pharos Programme (152 $7000014, \mathrm{R}-$ 263-000-B91-305); National Research Foundation (NRFCRP15-2015-03); National Natural Science Foundation of China (NSFC) (51222208, 51290273, 91433107); 
Youth 973 Program (2015CB932700); Priority Academic Program Development of Jiangsu Higher Education Institutions (PAPD); Collaborative Innovation Center of Suzhou Nano Science and Technology; Australian Research Council (ARC) (DE120101569, DP140101501, FT150100450).

Acknowledgment. This research was also partially supported by the National Research Foundation, Prime Minister's Office, Singapore under its Competitive Research Program. This work was performed in part at the Melbourne Centre for Nanofabrication (MCN) in the Victorian Node of the Australian National Fabrication Facility (ANFF).

†These authors contributed equally to this work.

\section{REFERENCES}

1. Q. Bao and K. P. Loh, "Graphene photonics, plasmonics, and broadband optoelectronic devices," ACS Nano 6, 3677-3694 (2012).

2. W. Gao, J. Shu, C. Qiu, and Q. Xu, "Excitation of plasmonic waves in graphene by guided-mode resonances," ACS Nano 6, 7806-7813 (2012).

3. A. Grigorenko, M. Polini, and K. Novoselov, "Graphene plasmonics," Nat. Photonics 6, 749-758 (2012).

4. Z. Fei, A. Rodin, G. Andreev, W. Bao, A. McLeod, M. Wagner, L. Zhang, Z. Zhao, M. Thiemens, and G. Dominguez, "Gate-tuning of graphene plasmons revealed by infrared nano-imaging," Nature 487, 82-85 (2012).

5. H. Yan, T. Low, W. Zhu, Y. Wu, M. Freitag, X. Li, F. Guinea, P. Avouris, and F. Xia, "Damping pathways of mid-infrared plasmons in graphene nanostructures," Nat. Photonics 7, 394-399 (2013).

6. L. Ju, B. Geng, J. Horng, C. Girit, M. Martin, Z. Hao, H. A. Bechtel, X. Liang, A. Zettl, and Y. R. Shen, "Graphene plasmonics for tunable terahertz metamaterials," Nat. Nanotechnol. 6, 630-634 (2011).

7. H. Yan, X. Li, B. Chandra, G. Tulevski, Y. Wu, M. Freitag, W. Zhu, P. Avouris, and F. Xia, "Tunable infrared plasmonic devices using graphene/insulator stacks," Nat. Nanotechnol. 7, 330-334 (2012).

8. K. Zhang, F. L. Yap, K. Li, C. T. Ng, L. J. Li, and K. P. Loh, "Large scale graphene/hexagonal boron nitride heterostructure for tunable plasmonics," Adv. Funct. Mater. 24, 731-738 (2014).

9. J. Zhang, C. Triola, and E. Rossi, "Proximity effect in graphenetopological-insulator heterostructures," Phys. Rev. Lett. 112, 096802 (2014).
10. S. Raghu, S. B. Chung, X.-L. Qi, and S.-C. Zhang, "Collective modes of a helical liquid," Phys. Rev. Lett. 104, 116401 (2010).

11. P. Di Pietro, M. Ortolani, O. Limaj, A. Di Gaspare, V. Giliberti, F. Giorgianni, M. Brahlek, N. Bansal, N. Koirala, and S. Oh, "Observation of Dirac plasmons in a topological insulator," Nat. Nanotechnol. 8, 556-560 (2013).

12. J. Christensen, A. Manjavacas, S. Thongrattanasiri, F. H. Koppens, and F. J. García de Abajo, "Graphene plasmon waveguiding and hybridization in individual and paired nanoribbons," ACS Nano 6, 431-440 (2012).

13. W. Dang, H. Peng, H. Li, P. Wang, and Z. Liu, "Epitaxial heterostructures of ultrathin topological insulator nanoplate and graphene," Nano Lett. 10, 2870-2876 (2010).

14. H. Qiao, J. Yuan, Z. Xu, C. Chen, S. Lin, Y. Wang, J. Song, Y. Liu, Q. Khan, H. Y. Hoh, C.-X. Pan, S. Li, and Q. Bao, "Broadband photodetectors based on graphene- $\mathrm{Bi}_{2} \mathrm{Te}_{3}$ heterostructure," ACS Nano 9, 1886-1894 (2015).

15. H. Mu, Z. Wang, J. Yuan, S. Xiao, C. Chen, Y. Chen, Y. Chen, J. Song, Y. Wang, Y. Xue, H. Zhang, and Q. Bao, "Graphene-Bi2Te ${ }_{3}$ heterostructure as saturable absorber for short pulse generation," ACS Photon. 2, 832-841 (2015)

16. S. Chen, L. Miao, X. Chen, Y. Chen, C. Zhao, S. Datta, Y. Li, Q. Bao, H. Zhang, Y. Liu, S. Wen, and D. Fan, "Few-layer topological insulator for all-optical signal processing using the nonlinear Kerr effect," Adv. Opt. Mater. 3, 1769-1778 (2015).

17. Y. L. Chen, Z. K. Liu, J. G. Analytis, J.-H. Chu, H. J. Zhang, B. H. Yan, S.-K. Mo, R. G. Moore, D. H. Lu, and I. R. Fisher, "Single Dirac cone topological surface state and unusual thermoelectric property of compounds from a new topological insulator family," Phys. Rev. Lett. 105, 266401 (2010).

18. Y. Xu, Z. Ren, W. Ren, G. Cao, K. Deng, and Y. Zhong, "Raman spectroscopy of two-dimensional $\mathrm{Bi}_{2} \mathrm{Te}_{x} \mathrm{Se}_{3-x}$ platelets produced by solvothermal method," Mater. Lett. 62, 4273-4276 (2008).

19. C. Wang, X. Zhu, L. Nilsson, J. Wen, G. Wang, X. Shan, Q. Zhang, S. Zhang, J. Jia, and Q. Xue, "In situ Raman spectroscopy of topological insulator $\mathrm{Bi}_{2} \mathrm{Te}_{3}$ films with varying thickness," Nano Res. 6, 688-692 (2013).

20. Y. Jiang, Y. Y. Sun, M. Chen, Y. Wang, Z. Li, C. Song, K. He, L. Wang, X. Chen, Q.-K. Xue, X. Ma, and S. B. Zhang, "Fermi-level tuning of epitaxial $\mathrm{Sb}_{2} \mathrm{Te}_{3}$ thin films on graphene by regulating intrinsic defects and substrate transfer doping," Phys. Rev. Lett. 108, 066809 (2013).

21. G. W. Hanson, "Dyadic green's functions and guided surface waves for a surface conductivity model of graphene," J. Appl. Phys. 103, 064302 (2013).

22. A. Zimmer, N. Stein, L. Johann, S. Van Gils, H. Terryn, E. Stijns, and C. Boulanger, "Infrared and visible dielectric function of electroplated $\mathrm{Bi}_{2 \pm x} \mathrm{Te}_{3 \pm x}$ films determined by spectroscopic ellipsometry," J. Electrochem. Soc. 152, G772-G777 (2005). 